\title{
El hospital en los tiempos del Covid-19: ingresos y mortalidad
}

\author{
Hospital in the time of Covid-19: hospital admissions and mortality
}

\author{
Beatriz García-Trincado ${ }^{1}$, José Conde Freire ${ }^{2}$, Manuel Francisco Liroa-Romero ${ }^{1}$ Ana Ferreiro-González ${ }^{3}$, Elena \\ Rodríguez-Ameijeiras ${ }^{1}$, Emilio Casariego-Vales ${ }^{1}$
}

${ }^{1}$ Servicio de Medicina Interna. Hospital Universitario Lucus Augusti. Lugo. ${ }^{2}$ Servicio de Documentación Clínica. Hospital Universitario Lucus Augusti. Lugo. ${ }^{3}$ Servicio de Medicina Intensiva. Hospital Universitario Lucus Augusti. Lugo.

\section{RESUMEN}

Objetivo: Describir la evolución de los ingresos hospitalarios, en plantas de hospitalización y Cuidados Intensivos, y la mortalidad en el hospital de un área afectada con tasas de incidencia medias por la pandemia COVID-19.

Métodos: Estudio observacional de todos los ingresos, y fallecimientos, que se produjeron entre los días 1 de marzo y 30 de abril de los años 2017, 2018, 2019 y 2020 en un hospital general. Consideramos las 00.00 horas del día 14 de marzo como inicio del estado de alarma y separación entre dos periodos: pre-estado de alarma (1-13 de marzo) y estado de alarma (14 de marzo-30 de abril).

Resultados: Comparando los meses de marzo-abril de los años 2017, 2018 y 2019 frente a 2020 se aprecia un descenso del número de ingresos: 23,33\% (Área Médica), 33,46\% (Área Quirúrgica) y 38,37\% en cuidados intensivos ( $p<0,0001$ en todos los casos). En las Áreas Médica y Quirúrgicas disminuyeron con el inicio del estado de alarma y en Cuidados Intensivos dos semanas más tarde. En el momento de máxima presión Ios pacientes con COVID-19 ocuparon un 9,8\% del total de camas del Centro. En las mismas fechas los fallecidos disminuyeron en las Áreas Médicas y Quirúrgicas un 3,98\% y 13,13\% respectivamente ( $p=N S$ ) y se incrementaron levemente (17,59\%; $p<0,001)$ en Cuidados Intensivos.

Conclusiones: Tras la instauración del estado de alarma, en un área sanitaria con incidencia media por la pandemia por COVID-19, se redujeron de forma significativa los ingresos hospitalarios sin apreciarse un exceso de mortalidad.

Palabras clave: Pandemia COVID-19, Mortalidad intrahospitalaria, Distribución hospitalaria

\section{INTRODUCCIÓN}

La pandemia por COVID-19 es la mayor emergencia de salud pública mundial de las últimas décadas. Su enorme alcance, la ausencia de tratamientos o vacunas disponibles, su elevada letalidad y su duración todavía incierta están provocando enormes presiones sobre el sistema sanitario ${ }^{1}$. La demanda que provoca de espacio hospitalario, personal o insumos y la obligada reorganización de servicios podría detraer recursos necesarios para atender otras enfermedades ${ }^{2}$. Sin duda la magnitud de la pandemia 0 las condiciones sanitarias basales en cada área son muy relevantes ${ }^{3}$. Sin embargo otros muchos factores como la declaración del estado de alarma, la sobrecarga informativa o la implantación de medidas de distanciamiento social modifican los comportamientos de la población y la demanda de asistencia sanitaria ${ }^{4}$.

La atención hospitalaria a las patologías habituales en los tiempos de pandemia es un tema poco analizado. Los análisis de otros episodios recientes se centran en las estrategias de control, los recursos precisos o los costes ${ }^{5-7}$. Por otra parte los estudios sobre la morbilidad y mortalidad que

\section{ABSTRACT}

Objective: To describe the evolution of the hospital admissions in the medical and surgical areas and intensive care units and that of the mortality in the hospital of an area affected with average incidence rates of the COVID-19 pandemic.

Methods: Observational study of all admissions in the medical services and deaths that were registered between March 1st and April 30th of years 2017, 2018, 2019 and 2020 in a General Hospital. We consider 00.00 on March 14th of 2020 as the beginning of the alarm state and as cut-off point between two periods: before the state of alert (March 1-13) and the state of alert (March 14- April 30)

Results: Comparing the months of March-April of years 2017, 2018, 2019 to 2020 a decrease in the number of admissions can be observed : 23,33\% (Medical area), 33,46\% (Surgical area) and 38,37\% (Intensive Care Units), ( $p<0,0001$ in every case). In the medical and surgical areas decreased with the beginning of state of alert's official statement and in Intensive Care Units two weeks after. During peak times patients with COVID-19 took up 9,8\% of total beds of the Center. At the same dates fatalities decreased in the medical and surgical areas 3,98\% and 13,13\% respectively $(p=N S)$ and increased slightly $(17,59 \% ; p<0,001)$ in Intensive Care Units.

Conclusions: After the state of alert's official statement in an healthcare area with average impact of COVID19 pandemic, hospital admissions dropped significantly without showing an excess in mortality.

Keywords: Covid 19 pandemic, in-hospital mortality, hospital management

se desencadenan tras diferentes tipos de catástrofes no han sido siempre concluyentes 0 aplicables. Por ello los efectos indirectos que causa una pandemia sobre la atención, la morbilidad o la mortalidad en otras patologías es poco conocida.

El objetivo de este estudio fue analizar cual fue la repercusión hospitalaria de la pandemia por COVID-19 en las primeras semanas de progresión del brote y tras la declaración del estado de alarma de alarma.

\section{PACIENTES Y MÉTODOS}

Estudio observacional de todos los ingresos en los servicios médicos, quirúrgicos y cuidados intensivos entre los días 1 de marzo y 30 de abril de los años 2017, 2018, 2019 y 2020 en el Hospital Universitario Lucus Augusti de Lugo. Este centro cubre la asistencia sanitaria de 240.000 habitantes y dispone de 879 camas.

La fuente de información fue el conjunto mínimo básico de datos del centro. Las variables analizadas, para cada día del 
periodo en estudio, fueron: número de ingresos, de fallecidos y altas en cada una de las áreas señaladas. En todos los casos la información recogida fue agrupada y sin acceso a ninguna historia clínica concreta, respetando la normativa vigente sobre la confidencialidad de los datos. Se decidió considerar las 00.00 horas del día 14 de marzo como el inicio del estado de alarma puesto que se anunció horas antes de su entrada en vigor y se entendió que ese era el momento en que comenzó a tener relevancia a los efectos de este estudio. Este momento es el punto de corte entre dos periodos: pre-estado de alarma (días 1 a 13 de marzo) y estado de alarma (días 14 a 30 de abril). El análisis del periodo del estado de alarma se realizó por quincenas. Todos los pacientes fueron seguidos hasta su alta o fallecimiento durante el mismo ingreso hospitalario.

En el análisis estadístico se utilizaron las técnicas descriptivas habituales. Se usó el test X2 para la comparación de variables cualitativas, con la corrección de Yates cuando fue preciso. En los cálculos de porcentajes se utilizó como referencia el número de ingresos en todos los casos. Para la comparación de 2 medias se utilizó, previa comprobación de la homocedasticidad, el test t de Student. En la comparación múltiple de medias se utilizó el análisis de la varianza y el test de Duncan. El nivel de significación estadística se estableció en $p<0,05$. Se utilizó el paquete estadístico SPSS versión 17.0 .

\section{RESULTADOS}

Entre el 1 de marzo y el 30 de abril, en las áreas hospitalarias de adultos señaladas, se ingresaron un total de 3377 pacientes en 2017, 3645 en 2018, 3601 en 2019 y 2614 en 2020. En la Tabla 1 se muestran los ingresos en los cuatro periodos de tiempo, antes y después del señalamiento del estado de alarma en marzo y por quincenas en abril, para los diferentes años en estudio en las distintas Áreas hospitalarias. El número de ingresos no fue estadísticamente diferente en el periodo de estudio de los años 2017, 2018 y 2019 ( $p=N S$ ). Sin embargo, cuando comparamos los años 2017, 2018 y 2019 frente a 2020 se aprecia un franco descenso del número de ingresos; $23,33 \%$ en el área médica, $33,46 \%$ en el área quirúrgica y del $38,37 \%$ en cuidados intensivos ( $p<0,0001$ en todos los casos). Se puede observar que los ingresos en las Áreas Médica y Quirúrgicas disminuyeron de manera significativa coincidiendo con el inicio del estado de alarma. No así los ingresos en Cuidados Intensivos que no se reducen hasta la primera quincena de abril. En el momento de máxima presión los pacientes con COVID-19 ocuparon un 9,8\% del total de las camas del Centro.

En la Tabla 2 se desglosan los fallecimientos intrahospitalarios en los mismos periodos de tiempo. Nuevamente, el número de fallecidos permaneció estable en el periodo de estudio en los años 2017, 2018 y 2019 (p=NS). Cuando comparamos los años 2017, 2018 y 2019 frente a 2020 se aprecia que los fallecidos disminuyeron en las áreas médicas y quirúrgicas un 3,98\% y 13,13\% respectivamente $(p=N S)$. Por el contrario en la Unidad de Cuidados Intensivos se incrementaron porcentualmente un 17,59\% $(p<0,001)$.

\section{DISCUSIÓN}

Este estudio muestra que en las primeras semanas de pandemia por COVID-19, y tras la declaración del estado de alarma, se redujeron de forma significativa los ingresos hospitalarios en las áreas médica, quirúrgica y de cuidados intensivos de un Hospital General. Por el contrario no se apreció un exceso de mortalidad durante el mismo periodo de tiempo.

Tabla 1. Distribución de ingresos hospitalarios entre 2017 y 2020.

\begin{tabular}{|l|c|c|c|c|c|}
\hline & $1-13$ MARZO & $14-31$ MARZ0 & $1-15$ ABRIL & $16-31$ ABRIL & TOTAL \\
\hline ÁREA MÉDICA & & & & & \\
Ingresos 2017-2019 (Media, DS) & $403,3(45,0)$ & $564(71,6)$ & $451,6(47,6)$ & $442(13,7)$ & 5976 \\
Ingresos 2020 $\left(n^{\circ}\right)$ & 473 & 414 & 383 & 346 & 1616 \\
\hline UCI & & & & & \\
Ingresos 2017-2019 (Media, DS) & $39,6(3,8)$ & $34,6(2,9)$ & $39,3(8,4)$ & $37,3(6,9)$ & 534 \\
Ingresos 2020 $\left(n^{\circ}\right)$ & 23 & 43 & 22 & 32 & 120 \\
\hline ÁREA QUIRÚRGICA & & & & \\
Ingresos 2017-2019 (Media, DS) & $283,3(43,1)$ & $348,3(35,8)$ & $334(39,3)$ & $331,6(0,9)$ & 4113 \\
Ingresos 2020 $\left(n^{\circ}\right)$ & 334 & 187 & 156 & 201 & 878 \\
\hline
\end{tabular}

Tabla 2. Mortalidad hospitalaria entre 2017 y 2020.

\begin{tabular}{|c|c|c|c|c|c|}
\hline & 1-13 MARZO & 14-31 MARZ0 & 1-15 ABRIL & 16-31 ABRIL & TOTAL \\
\hline \multicolumn{6}{|l|}{ ÁREA MÉDICA } \\
\hline Fallecidos 2017-2019 (Media, DS) & $47,3(3,2)$ & $57,1(3,5)$ & $44,1(2,1)$ & $48(0,8)$ & 589 \\
\hline Fallecidos $2020\left(n^{0}\right)$ & 53 & 61 & 42 & 35 & 191 \\
\hline \multicolumn{6}{|l|}{$\mathrm{UCl}$} \\
\hline Fallecidos 2017-2019 (Media, DS) & $5,66(3,7)$ & $8(3,7)$ & $5,7(3,1)$ & $5,3(1,2)$ & 74 \\
\hline Fallecidos $2020\left(n^{0}\right)$ & 5 & 10 & 7 & 7 & 29 \\
\hline \multicolumn{6}{|l|}{ ÁREA QUIRÚRGICA } \\
\hline Fallecidos 2017-2019 (Media, DS) & $3,3(1,9)$ & $6(2,1)$ & $6,3(0,9)$ & $6(0,8)$ & 59 \\
\hline Fallecidos $2020\left(n^{0}\right)$ & 5 & 3 & 6 & 3 & 17 \\
\hline
\end{tabular}


La pandemia por COVID-19 ha afectado profundamente a los hospitales por las dificultades de atender a un gran número de pacientes con enfermedad severa en un corto espacio de tiempo, por la ausencia de tratamientos específicos o por no disponer de las medidas de protección precisas ${ }^{4,9,10}$. Los datos de un gran hospital español en Madrid, donde la epidemia ha tenido una tasa de incidencia elevada, muestran como el COVID-19 ocupó la práctica totalidad del centro, el $10 \%$ de los ingresados requirió tratamiento en cuidados intensivos y la mortalidad fue ligeramente superior al $20 \%{ }^{10}$. En nuestro hospital, en un área con una tasa de incidencia media ${ }^{11}$, la situación ha sido muy distinta. De hecho la ocupación máxima por COVID-19 apenas alcanzó el 10\% de las camas del hospital. Entendemos que esta baja ocupación debe explicarse por la suma de varias circunstancias concomitantes. En primer lugar, y sobre todo, fruto del éxito de las medidas de confinamiento social. A pesar de su importancia creemos que este hecho no basta para explicar la reducción de ingresos en todas las áreas de adultos del Centro. En segundo lugar, es evidente que la suspensión temporal de las cirugías programadas justifica, al menos parcialmente, la menor ocupación de los servicios quirúrgicos. En tercer lugar la disminución de la afluencia a urgencias, de hasta un $65,1 \%$, tras la declaración del estado de alarma ${ }^{12}$. A nuestro entender el menor uso del servicio de Urgencias tiene, su vez, varias razones que incluyen desde la reducción de traumatismos por la baja movilidad durante el estado de alarma hasta a las reticencias de la población a acudir al hospital cuando percibe que es una zona de riesgo de contagio de una enfermedad grave.

A pesar de que el COVID-19 es una enfermedad con elevadas tasas de letalidad ${ }^{10}$, en nuestro hospital durante los 2 meses en estudio no se apreció un exceso de mortalidad (Tabla 2). La disminución de la ocupación hospitalaria se acompañó de una leve reducción de la mortalidad en las plantas de hospitalización y un pequeño, aunque significativo, aumento en Cuidados Intensivos. La interpretación de estos datos es compleja tanto por el escaso número de fallecidos como por la acusada reducción del número de ingresos. Consideramos que una explicación adecuada es que se mantiene la mortalidad esperable relacionada con las patologías más graves en un contexto de reducción de ingresos por motivos menos graves.

Este estudio debe valorarse teniendo en cuenta sus limitaciones. En primer lugar son datos de un único hospital y quizá no sean similares a los de otros Centros de nuestro entorno. Si bien esto es cierto, nuestro estudio alerta sobre las peculiaridades de la atención en estas situaciones y puede ser útil para diseñar nuevos y más amplios estudios que confirmen estos datos y que ayuden en el control de crisis futuras. Una segunda cuestión es que este estudio se diseñó para analizar aspectos asistenciales al inicio de la pandemia y no fases más tardías. Por lo tanto no es útil más allá de los dos primeros meses de crisis. En tercer lugar utilizamos fechas de referencia que se corresponden con meses natu- rales, que no se relacionan con el curso natural de la enfermedad. La razón es que el objetivo del estudio es conocer situación en los primeras semanas tras la declaración del estado de alarma. Esta elección permite disponer de una referencia, clara y sencilla (antes y después de la declaración) y ser útil tanto en el diseño y como comparable con los resultados de futuros estudios. Por último, en nuestra área las tasas de incidencia de la pandemia fueron intermedias entre las registradas en las distintas Comunidades Autónomas. Por tanto nuestras cifras pudiesen no ser extrapolables a lugares con tasas de incidencia extremadamente altas 0 bajas.

En RESUMEN, en las primeras 6 semanas tras la instauración del estado de alarma, en un área sanitaria con incidencia media por la pandemia por COVID-19, se redujeron de forma significativa los ingresos hospitalarios y no se apreció un exceso de mortalidad. Son precisos nuevos estudios que confirmen estos resultados en otras áreas geográficas y en plazos temporales más amplios.

\section{CONFLICTO DE INTERESES}

Los autores declaran no tener ningún conflicto de intereses.

\section{BIBLIOGRAFÍA}

1. Ministerio de Sanidad. Centro de Coordinación de Alertas y Emergencias Sanitaria. Actualización n 99 . Enfermedad por el coronavirus (COVID-19). Disponible en: https://www.mscbs.gob.es/profesionales/saludPublica/ccayes/alertasActual/nCovChina/documentos/Actualizacion_99_COVID-19.pdf. Consultado el 9 de mayo de 2020.

2. Søreide K, Hallet J, Matthews JB, Schnitzbauer AA, Line PD, Lai PBS, et al. Immediate and Long-Term Impact of the COVID-19 Pandemic on Delivery of Surgical Services. Br J Surg. 2020;107(10):1250-1261.

3. Bedford J, Enria D, Giesecke J, Heymann DL, Ihekweazu C, Kobinger G, et al ; WHO Strategic and Technical Advisory Group for Infectious Hazards. COVID-19: Towards Controlling of a Pandemic. Lancet. 2020;395(10229):1015-1018.

4. Legido-Quigley H, Mateos-García JT, Campos VR, Gea-Sánchez M, Muntaner C, McKee M. The Resilience of the Spanish Health System Against the COVID-19 Pandemic. Lancet Public Health. 2020;5(5):e251-e252.

5. Fineberg H. Pandemic preparedness and responde-Lesson form the H1N1 influenza in 2009. N Eng J med. 2014; 370: 1335-42.

6. Lau K, Hauck K, Miraldo M. Excess influenza hospital admissions and costs due to the 2009 H1N1 pandemic in England. Health Economics. 2019; 28: 175-188.

7. Saunders-Hastings P, Crispo JAG, Sikora L, Krewski D. Effectiveness of Personal Protective Measures in Reducing Pandemic Influenza Transmission: A Systematic Review and Meta-Analysis. Epidemics. 2017 Sep;20:1-20.

8. Chi JS, Speakman MT, Poole WK, Kandefer SC, Kloner RA. Hospital Admissions for Cardiac Events in New York City After September 11, 2001. Am J Cardiol. 2003;92(1):61-3.

9. Comunidad de Madrid. La Comunidad de Madrid aprueba medidas extraordinarias por el coronavirus. 2020. https://www.comunidad.madrid/sites/defaultfiles/doc/sanidad/ nota_de_prensa_cm_09.03.2020_21h.pdf (Consultado el 9 de mayo de 2020).

10. Borobia AM, Carcas AJ, Arnalich F, Álvarez-Sala R, Montserrat J, Quintana M. for the COVID@HULP Working Group. A cohort of patients with COVID-19 in a major teaching hospital in Europe. Disponible en: https://www.fesemi.org/sites/default/ files/documentos/560.pdf. Consultado el 9 de mayo de 2020.

11. Ministerio de Sanidad. Centro de Coordinación de Alertas y Emergencias Sanitaria Actualización nº 68. Enfermedad por el coronavirus (COVID-19). https://www. mscbs.gob.es/profesionales/saludPublica/ccayes/alertasActual/nCov-China/ documentos/Actualizacion_68_COVID-19.pdf. Consultado el 10 de mayo de 2020.

12. Conde-Freire J, Pérez López A, Rodríguez-Álvarez A, Gil-Mouce C, Rodríguez Ameijeiras E, Liroa-Romera MF et al. Pandemia por covid19: otras consecuencias Galicia Clin. 2021; 82-1: 5-8 